\title{
Analysis of an ambulatory care pathway for patients with COVID-19 utilising remote pulse oximetry
}

\author{
Authors: Jonathon Kyriakides, ${ }^{\mathrm{A}}$ Aria Khani, ${ }^{\mathrm{A}}$ Charlotte Kelly ${ }^{\mathrm{A}}$ and Reginald Coleman ${ }^{\mathrm{A}}$
}

\section{Introduction}

During the COVID-19 pandemic, trusts across London aimed to discharge COVID-19 patients with oxygen saturations $\left(\mathrm{SaO}_{2}\right)$ of 90-94\%, due to concerns regarding the limited hospital resource of both inpatient beds and supplemental oxygen. However, the safe discharge of these patients from the emergency department (ED) is difficult due to uncertainties surrounding the trajectory of the disease course and the infectivity of the disease limiting follow-up options. A novel pathway consisting of home pulse oximetry with telephone follow-up was created to facilitate safe discharge from the ED of a London district general hospital. The primary objective was to utilise home pulse oximetry to prevent hospital admission. The secondary objective was to identify those requiring further care or investigation.

\section{Methods}

Adult patients with confirmed or suspected COVID-19 with an $\mathrm{SaO}_{2}$ of $90-94 \%$ who were otherwise suitable for discharge were identified. These patients were discharged from the ED with a pulse oximeter. $\mathrm{SaO}_{2}$ was measured three times a day for 7 days. Patients received a structured telephone consultation on days 2 , 5 and 7 post-discharge from the ED, and a decision was made as to whether further clinical assessment in the ED was necessary. Patients were asked to return to the ED based on standard safety netting information or if their $\mathrm{SaO}_{2}$ measured below $90 \%$ at rest on two or more occasions within a 24 -hour period.

\section{Results and discussion}

Of the 20 patients discharged on the pathway, $85 \%$ avoided hospital admission, while $15 \%$ were reassessed and subsequently admitted to hospital. $20 \%$ of patients required reassessment in the ED but did not require admission (Fig 1).

The mean age of patients who were admitted following reassessment was 65 , compared with a mean age of 54 in the original cohort of patients. This is in keeping with increasing age being a poor prognostic factor in COVID-19. ${ }^{1}$

All patients who required reassessment had at least one comorbidity, with three patients having two or more

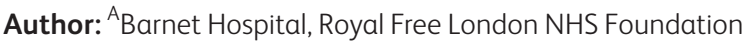
Trust, London, UK

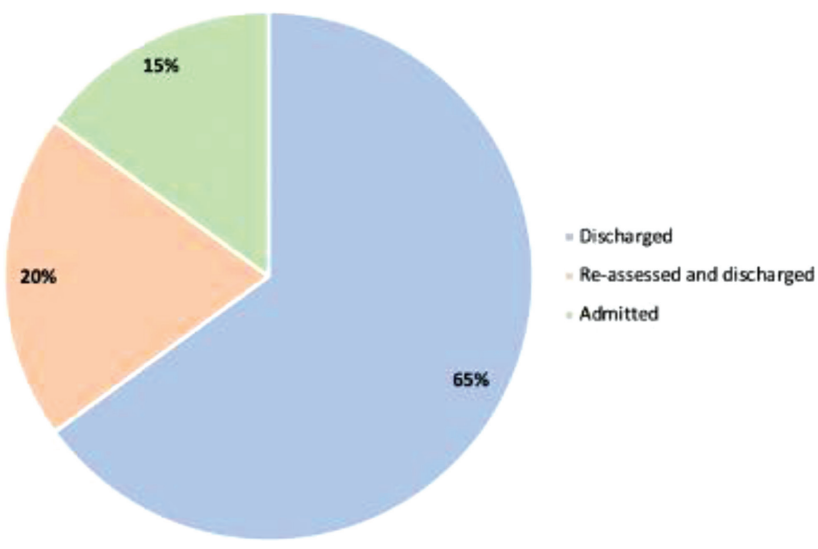

Fig 1. Patient outcomes.

comorbidities. Hypertension was more common in the cohort of patients who required reassessment, which supports previous studies showing that cardiovascular comorbidities are associated with increased mortality in COVID-19. ${ }^{2}$

Pulmonary infiltrates on chest X-ray were more common in the cohort of patients who required reassessment, mirroring the importance of radiological findings in the outcomes of COVID-19 patients. $^{3}$

\section{Conclusions}

Home pulse oximetry with telephone follow-up was used to prevent hospital admission in a considerable proportion of patients who would have otherwise been admitted in the absence of this pathway. Telephone follow-up effectively identified patients who required further clinical assessment. This study demonstrates the potential for safe ambulation of a subgroup of patients with COVID-19, while identifying practical inclusion criteria that could be replicated in ambulatory units across the UK. Future research should focus on the inclusion of various prognostic markers, due to their potential power in determining disease trajectory.

\section{Conflicts of interest}

None declared. 


\section{References}

1 Banerjee A, Pasea L, Harris S et al. Estimating excess 1-year mortality associated with the COVID-19 pandemic according to underlying conditions and age: a population-based cohort study. Lancet 2020;395:1715-25.
2 Chen $\mathrm{T}, \mathrm{Wu} \mathrm{D}$, Chen $\mathrm{H}$ et al. Clinical characteristics of 113 deceased patients with coronavirus disease 2019: retrospective study. BMJ 2020;368:m1091.

3 Borghesi A, Maroldi R. COVID-19 outbreak in Italy: experimental chest X-ray scoring system for quantifying and monitoring disease progression. Radiol Med 2020;125:509-13. 